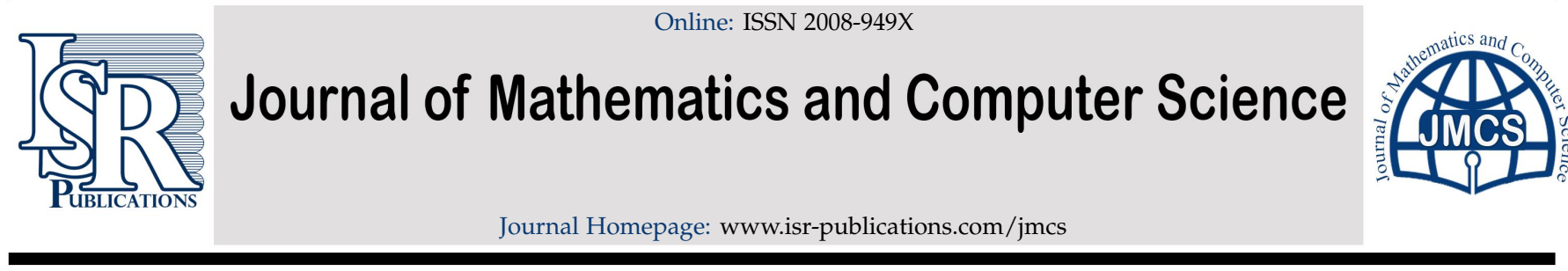

\title{
Extended cubic B-spline method for solving a system of non- linear second-order boundary value problems
}

\author{
Ahmed Salem Heilat ${ }^{\mathrm{a}, *}$, Reyadh Salem Hailat ${ }^{\mathrm{b}}$ \\ ${ }^{a}$ Department of Mathematics, Jadara University, P. O. Box (733), 21111 Irbid, Jordan. \\ ${ }^{b}$ PAAET Basic College, Rd No 103, Al Kuwayt, Kuwait.
}

\begin{abstract}
In this paper, we develop the extended cubic B-spline method for solving a system of nonlinear second-order boundary value problems. A quasilinearization approach is used and the error estimate is obtained. The accuracy of the method depends on two free parameters. We apply the method on four examples and the results which obtained using extended cubic B-spline indicate that the method is efficient and accurate.
\end{abstract}

Keywords: Boundary value problems, quasilinearization, extended cubic B-spline.

2020 MSC: 34A34, 65L10.

(C)2020 All rights reserved.

\section{Introduction}

In this study, we consider the following system of nonlinear second-order boundary value problems:

$$
\left\{\begin{array}{l}
u^{\prime \prime}(x)+a_{1}(x) u^{\prime}(x)+a_{2}(x) u(x)+a_{3}(x) v^{\prime \prime}(x)+a_{4}(x) v^{\prime}(x)+a_{5}(x) v(x)+G_{1}(x, u, v)=f_{1}(x), \\
v^{\prime \prime}(x)+b_{1}(x) v^{\prime}(x)+b_{2}(x) v(x)+b_{3}(x) u^{\prime \prime}(x)+b_{4}(x) u^{\prime}(x)+b_{5}(x) u(x)+G_{2}(x, u, v)=f_{2}(x), \\
u(0)=u(1)=0, v(0)=v(1)=0,
\end{array}\right.
$$

where $0 \leqslant x \leqslant 1, f_{1}(x)$ and $f_{2}(x)$ are continuous functions, $G_{1}$ and $G_{2}$ are nonlinear functions of $u$ and $v$. Also $a_{i}(x)$ and $b_{i}(x), i=1,2,3,4,5$ are real-valued functions of $x$ that are sufficiently smooth. System of nonlinear boundary value problems involving ordinary differential equations arise in real life applications and the existence of solutions to such system was discussed in $[7,8,28]$.

Various approximate methods have been developed and these include He's homotopy perturbation method [25], Laplace homotopy analysis method [23], variational iteration method [21], homotopy perturbation-reproducing kernel method [14], reproducing kernel method [13], sinc-collocation method [11, 12], local radial basis function based differential quadrature [10], Chebyshev finite difference [26],

\footnotetext{
${ }^{*}$ Corresponding author

Email address: ahmed_heilat@yahoo.com (Ahmed Salem Heilat)

doi: $10.22436 /$ jmcs.021.03.06
}

Received: 2020-02-09 Revised: 2020-02-23 Accepted: 2020-03-03 
continuous genetic algorithm [2], modified homotopy analysis method [5], three positive solution method [19], spline collocation approach [20], cubic B-spline scaling functions [9], Chebyshev wavelet finite difference [22], B-spline method [6], multidimensional triangular models [16], multistage optimal homotopy asymptotic method [3, 4], and hybrid cubic B-spline method [18]. The extended cubic B-spline was first proposed by Han and Liu in 2003 [27]. It involved one free parameter, $\lambda$. This parameter was found to increase the flexibility of the spline curve. The cubic B-spline was developed to obtain an extended cubic B-spline with degree 4, 5, and 6 in [29]. Extended cubic B-spline method (ECBSM) was developed to solve linear and singular two point boundary value problems involving ordinary differential equations in $[1,15]$. The same approach was also used to solve a linear system of second order boundary value problems by the present authors [17]. Two free parameters were optimized to obtain accurate results.

In this paper, we extend the use of ECBSM to solve system of nonlinear second order boundary value problems involving ordinary differential equations. The organization of this paper is as follows. The quasilinearization technique to handle the nonlinearity is discussed in Section 2. The extended cubic B-spline method is developed and the solution of the system is discussed in Section 3. The error estimate is obtained in Section 4. Finally, Section 5 presents four numerical examples.

\section{Quasilinearization}

The quasilinearization method is utilized to reduce the given nonlinear problem (1.1) to a sequence of linear problems. The method used is adopted from [1, 15]. From (1.1), we have

$$
\left\{\begin{array}{l}
G_{1}\left(x, u, v, u_{x}, v_{x}\right)=L_{1}\left(x, u, v, u_{x}, v_{x}\right)+f_{1}(x) \\
G_{2}\left(x, u, v, u_{x}, v_{x}\right)=L_{2}\left(x, u, v, u_{x}, v_{x}\right)+f_{2}(x)
\end{array}\right.
$$

where

$$
\left\{\begin{array}{l}
\mathrm{L}_{1}\left(x, u, v, u_{x}, v_{x}\right)=-u^{\prime \prime}(x)-a_{1}(x) u^{\prime}(x)-a_{2}(x) u(x)-a_{3}(x) v^{\prime \prime}(x)-a_{4}(x) v^{\prime}(x)-a_{5}(x) v(x) \\
L_{2}\left(x, u, v, u_{x}, v_{x}\right)=-v^{\prime \prime}(x)-b_{1}(x) v^{\prime}(x)-b_{2}(x) v(x)-b_{3}(x) u^{\prime \prime}(x)-b_{4}(x) u^{\prime}(x)-b_{5}(x) u(x)
\end{array}\right.
$$

Initial approximations for the functions $u(x)$ and $v(x)$ in $G_{1}\left(x, u, v, u_{x}, v_{x}\right)$ and $G_{2}\left(x, u, v, u_{x}, v_{x}\right)$, denoted by $u^{(0)}(x)=c_{1}$ and $v^{(0)}(x)=c_{3}$, are chosen. $G_{1}\left(x, u, v, u_{x}, v_{x}\right)$ and $G_{2}\left(x, u, v, u_{x}, v_{x}\right)$ are then expanded about these approximations, to obtain

$$
\left\{\begin{array}{c}
\mathrm{G}_{1}\left(x, u^{(1)}, u_{x}^{(1)}, v^{(1)}, v_{x}^{(1)}\right)=G_{1}\left(x, u^{(0)}, u_{x}^{(0)}, v^{(0)}, v_{x}^{(0)}\right)+\left(u^{(1)}-u^{(0)}\right)\left(\frac{\partial G_{1}}{\partial u}\right)_{\left(x, u^{(0)}\right)} \\
+\left(u_{x}^{(1)}-u_{x}^{(0)}\right)\left(\frac{\partial G_{1}}{\partial u_{x}}\right)_{\left(x, u_{x}^{(0)}\right)}+\left(v^{(1)}-v^{(0)}\right)\left(\frac{\partial G_{1}}{\partial v}\right)_{\left(x, v^{(0)}\right)}+\left(v_{x}^{(1)}-v_{x}^{(0)}\right)\left(\frac{\partial G_{1}}{\partial v_{x}}\right)_{\left(x, v_{x}^{(0)}\right)^{\prime}}, \\
G_{2}\left(x, u^{(1)}, u_{x}^{(1)}, v^{(1)}, v_{x}^{(1)}\right)=G_{2}\left(x, u^{(0)}, u_{x}^{(0)}, v^{(0)}, v_{x}^{(0)}\right)+\left(u^{(1)}-u^{(0)}\right)\left(\frac{\partial G_{2}}{\partial u}\right)_{\left(x, u^{(0)}\right)}{ }^{(0)}\left(u_{x}^{(1)}-u_{x}^{(0)}\right)\left(\frac{\partial G_{2}}{\partial u_{x}}\right)_{\left(x, u_{x}^{(0)}\right)}+\left(v^{(1)}-v^{(0)}\right)\left(\frac{\partial G_{2}}{\partial v}\right)_{\left(x, v^{(0)}\right)}+\left(v_{x}^{(1)}-v_{x}^{(x)}\right)\left(\frac{\partial v_{x}}{\left.\partial x, v_{x}^{(0)}\right)} .\right.
\end{array}\right.
$$

Generally for $s=0,1,2, \ldots(s=$ iteration index $)$, we can write

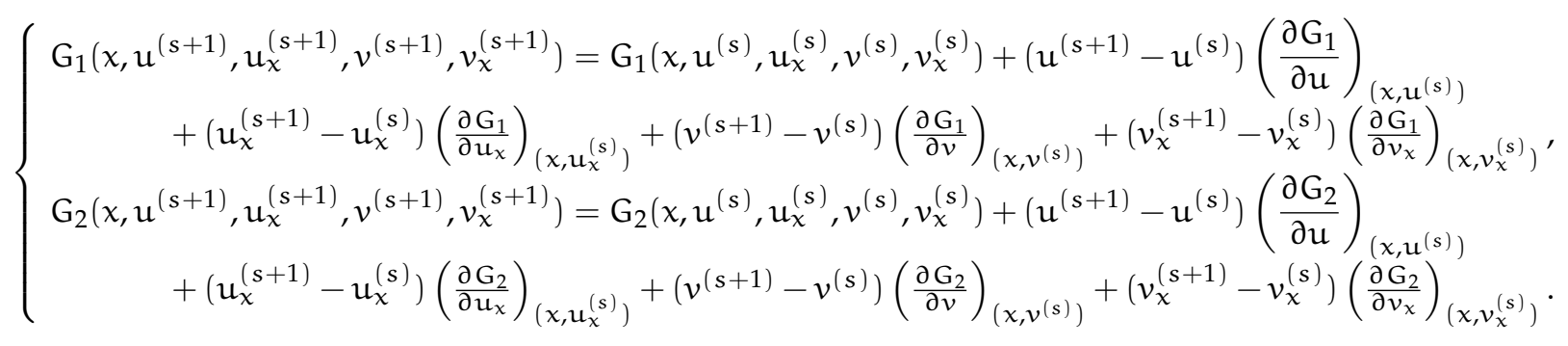


Here, we use Taylor's expansion. By substituting (2.1) into the expansion (2.2), the following sequence of linear equations are resulted:

$$
\left\{\begin{array}{c}
\mathrm{L}_{1}\left(x, u^{(s+1)}, u_{x}^{(s+1)}, v^{(s+1)}, v_{x}^{(s+1)}\right) \cong L_{1}\left(x, u^{(s)}, u_{x}^{(s)}, v^{(s)}, v_{x}^{(s)}\right)+\left(u^{(s+1)}-u^{(s)}\right)\left(\frac{\partial G_{1}}{\partial u}\right)_{\left(x, u^{(s)}\right)} \\
\quad+\left(u_{x}^{(s+1)}-u_{x}^{(s)}\right)\left(\frac{\partial G_{1}}{\partial u_{x}}\right)_{\left(x, u_{x}^{(s)}\right)}+\left(v^{(s+1)}-v^{(s)}\right)\left(\frac{\partial G_{1}}{\partial v}\right)_{\left(x, v^{(s)}\right)}+\left(v_{x}^{(s+1)}-v_{x}^{(s)}\right)\left(\frac{\partial G_{1}}{\partial v_{x}}\right)_{\left(x, v_{x}^{(s)}\right)^{\prime}} \\
\mathrm{L}_{2}\left(x, u^{(s+1)}, u_{x}^{(s+1)}, v^{(s+1)}, v_{x}^{(s+1)}\right) \cong L_{2}\left(x, u^{(s)}, u_{x}^{(s)}, v^{(s)}, v_{x}^{(s)}\right)+\left(u^{(s+1)}-u^{(s)}\right)\left(\frac{\partial G_{1}}{\partial u}\right)_{\left(x, u^{(s)}\right)} \\
\quad+\left(u_{x}^{(s+1)}-u_{x}^{(s)}\right)\left(\frac{\partial G_{1}}{\partial u_{x}}\right)_{\left(x, u_{x}^{(s)}\right)}+\left(v^{(s+1)}-v^{(s)}\right)\left(\frac{\partial G_{1}}{\partial v}\right)_{\left(x, v^{(s)}\right)}+\left(v_{x}^{(s+1)}-v_{x}^{(s)}\right)\left(\frac{\partial G_{1}}{\partial v_{x}}\right)_{\left(x, v_{x}^{(s)}\right)}
\end{array}\right.
$$

We can compute $\left(\frac{\partial G_{1}}{\partial u^{\prime}}\right)_{\left(x, u^{(s)}\right)^{\prime}}\left(\frac{\partial G_{1}}{\partial u_{x}}\right)_{\left(x, u_{x}^{(s)}\right)^{\prime}}\left(\frac{\partial G_{1}}{\partial v}\right)_{\left(x, v^{(s)}\right)^{\prime}}$ and $\left(\frac{\partial G_{1}}{\partial v_{x}}\right)_{\left(x, v_{x}^{(s)}\right)^{\prime}}$ respectively. The same technique can be used to compute the derivative of $\mathrm{G}_{2}$. Equation (2.3) can be written as follows.

$$
\left\{\begin{array}{c}
u_{x x}^{(s+1)}(x)+a_{1}^{(s)}(x) u_{x}^{(s+1)}(x)+a_{2}^{(s)}(x) u^{(s+1)}(x)+a_{3}^{(s)}(x) v_{x x}^{(s+1)}(x) \\
\quad+a_{4}^{(s)}(x) v_{x}^{(s+1)}(x)+a_{5}^{(s)}(x) v^{(s+1)}(x)=f_{1}^{(s)}(x) \\
v_{x x}^{(s+1)}(x)+b_{1}^{(s)}(x) v_{x}^{(s+1)}(x)+b_{2}^{(s)}(x) v^{(s+1)}(x)+b_{3}^{(s)}(x) u_{x x}^{(s+1)}(x) \\
+b_{4}^{(s)}(x) u_{x}^{(s+1)}(x)+b_{5}^{(s)}(x) u^{(s+1)}(x)=f_{2}^{(s)}(x)
\end{array}\right.
$$

with boundary conditions

$$
\begin{cases}u^{(s+1)}(0)=\alpha, & u^{(s+1)}(1)=\beta, \\ v^{(s+1)}(0)=\gamma, & v^{(s+1)}(1)=\delta .\end{cases}
$$

To solve the sequence of linear equations BVPs given in (2.4) with boundary conditions (2.5), we apply the ECBS method. Consider a partition $\pi$ of $[a, b]$ as equally-spaced knots $x_{i}$ into $n$ segments $\left[x_{i}, x_{i+1}\right]$, $i=0,1, \ldots, n$, where $h=\frac{b-a}{n}$ such that $x_{i}=x_{0}+i h, i \in \mathbb{Z}$. Suppose that $U\left(x, \lambda_{1}\right)$ and $V\left(x, \lambda_{1}\right)$ be ECBS solution to (2.4), satisfying the interpolating conditions. Then the ECBS approximations for $\mathrm{U}\left(\mathrm{x}, \lambda_{1}\right)$, $u^{\prime}\left(x, \lambda_{1}\right), u^{\prime \prime}\left(x, \lambda_{1}\right), v\left(x, \lambda_{2}\right), v^{\prime}\left(x, \lambda_{2}\right)$, and $v^{\prime \prime}\left(x, \lambda_{2}\right)$ at $i^{\text {th }}$ knots. Discretizing (2.4) at $x_{i}$, we obtain

$$
\left\{\begin{array}{l}
u_{x x}^{(s+1)}\left(x_{i}\right)+a_{1}^{(s)}\left(x_{i}\right) u_{x}^{(s+1)}\left(x_{i}\right)+a_{2}^{(s)}\left(x_{i}\right) u^{(s+1)}\left(x_{i}\right)+a_{3}^{(s)}\left(x_{i}\right) V_{x x}^{(s+1)}\left(x_{i}\right) \\
\quad+a_{4}^{(s)}\left(x_{i}\right) V_{x}^{(s+1)}\left(x_{i}\right)+a_{5}^{(s)}\left(x_{i}\right) V^{(s+1)}\left(x_{i}\right)=f_{1}^{(s)}\left(x_{i}\right), \\
v_{x x}^{(s+1)}\left(x_{i}\right)+b_{1}^{(s)}\left(x_{i}\right) V_{x}^{(s+1)}\left(x_{i}\right)+b_{2}^{(s)}\left(x_{i}\right) V^{(s+1)}\left(x_{i}\right)+b_{3}^{(s)}\left(x_{i}\right) u_{x x}^{(s+1)}\left(x_{i}\right) \\
\quad+b_{4}^{(s)}\left(x_{i}\right) u_{x}^{(s+1)}\left(x_{i}\right)+b_{5}^{(s)}\left(x_{i}\right) U^{(s+1)}\left(x_{i}\right)=f_{2}^{(s)}\left(x_{i}\right) .
\end{array}\right.
$$

Substituting $U\left(x, \lambda_{1}\right), u^{\prime}\left(x, \lambda_{1}\right), u^{\prime \prime}\left(x, \lambda_{1}\right), v\left(x, \lambda_{2}\right), v^{\prime}\left(x, \lambda_{2}\right)$, and $v^{\prime \prime}\left(x, \lambda_{2}\right)$ into equation

$$
\left\{\begin{array}{l}
\mathrm{U}\left(x, \lambda_{1}\right)=\sum_{i=-3}^{n-1} \alpha_{i} E_{i}^{4}\left(x, \lambda_{1}\right), x \in\left[x_{0}, x_{n}\right], \alpha_{i} \in \mathbb{R}, \\
V\left(x, \lambda_{2}\right)=\sum_{i=-3}^{n-1} \beta_{i} E_{i}^{4}\left(x, \lambda_{2}\right), x \in\left[x_{0}, x_{n}\right], \beta_{i} \in \mathbb{R},
\end{array}\right.
$$

we get a system of $2(n+1)$ equations in $2(n+3)$ unknowns. Therefore, we still need two more equations, which are obtained from the boundary conditions (2.5) as

$$
\begin{cases}\mathrm{u}^{(s+1)}(0)=\alpha, & \mathrm{u}^{(s+1)}(1)=\beta, \\ \mathrm{v}^{(s+1)}(0)=\gamma, & \mathrm{v}^{(s+1)}(1)=\delta .\end{cases}
$$

The iterations can be stopped by setting the prescribed absolute error criterion. For any $\epsilon>0$, $\left|u_{i}^{(s+1)}(x)-u_{i}^{(s)}(x)\right| \leqslant \epsilon$ and $\left|v_{i}^{(s+1)}(x)-v_{i}^{(s)}(x)\right| \leqslant \epsilon$, where $\epsilon$ must be chosen small enough, for all $i$ that appears. In this study, the value of $\epsilon$ is taken to be $10^{-7}$ as used by [24]. 


\section{Extended cubic B-spline method}

After quasilinearization, we consider equations (2.6) and (2.8), system of linear second order two-point boundary value problems, where $a \leqslant x \leqslant b, f_{1}(x)$ and $f_{2}(x)$ are continuous functions, and $a_{i}(x)$ and $b_{i}(x)$ for $i=1,2,3,4,5$ are real-valued functions of $x$ that are sufficiently smooth.

In this section, the ECBSM is developed for solving a system of nonlinear second order boundary value problems (1.1). Consider a partition $\pi$ of $[a, b]$ of equally-spaced knots $x_{i}$ with $n$ segments, $\left[x_{i}, x_{i+1}\right]$, for $i=0,1, \ldots, n$, where $a=x_{0}<x_{1}<\cdots<x_{n}=b$, such that

$$
\mathrm{h}=\frac{\mathrm{b}-\mathrm{a}}{\mathrm{n}}, \quad \mathrm{x}_{0}=\mathrm{a}, \quad \mathrm{x}_{\mathrm{i}}=\mathrm{x}_{0}+\mathrm{ih}, \quad \mathrm{i} \in \mathbb{Z} .
$$

The approximate solution $\mathrm{U}\left(x, \lambda_{1}\right)$ and $\mathrm{V}\left(x, \lambda_{2}\right)$ to the exact solutions $\mathrm{u}(\mathrm{x})$ and $v(x)$, respectively, are given by equation (2.7). The extended cubic B-spline basis is given by [1]

$$
E_{i}^{4}(x, \lambda)=\frac{1}{24 h^{4}} \begin{cases}4 h(1-\lambda)\left(x-x_{i}\right)^{3}+3 \lambda\left(x-x_{i}\right)^{4}, & x \in\left[x_{i}, x_{i+1}\right], \\ (4-\lambda) h^{4}+12 h^{3}\left(x-x_{i+1}\right)+6 h^{2}(2+\lambda)\left(x-x_{i+1}\right)^{2} & \\ -12 h\left(x-x_{i+1}\right)^{3}-3 \lambda\left(x-x_{i+1}\right)^{4}, & x \in\left[x_{i+1}, x_{i+2}\right], \\ (4-\lambda) h^{4}+12 h^{3}\left(x_{i+3}-x\right)+6 h^{2}(2+\lambda)\left(x_{i+3}-x\right)^{2} & \\ -12 h\left(x_{i+3}-x\right)^{3}-3 \lambda\left(x_{i+3}-x\right)^{4}, & x \in\left[x_{i+2}, x_{i+3}\right], \\ 4 h(1-\lambda)\left(x_{i+4}-x\right)^{3}+3 \lambda\left(x_{i+4}-x\right)^{4}, & x \in\left[x_{i+3}, x_{i+4}\right] .\end{cases}
$$

The values of $E_{i}, E_{i}^{\prime}$, and $E_{i}^{\prime \prime}$ at the nodal points are given in Table 1 .

Table 1: Coefficient of $E_{i}, E_{i}^{\prime}$, and $E_{i}^{\prime \prime}$.

\begin{tabular}{cccccc}
\hline$\chi$ & $x_{i}$ & $x_{i+1}$ & $\chi_{i+2}$ & $x_{i+3}$ & $x_{i+4}$ \\
\hline$E_{i}$ & 0 & $\frac{4-\lambda}{24}$ & $\frac{8+\lambda}{12}$ & $\frac{4-\lambda}{24}$ & 0 \\
$E_{i}^{\prime}$ & 0 & $\frac{-1}{2 h}$ & $\frac{0}{h}$ & $\frac{1}{2 h}$ & 0 \\
$E_{i}^{\prime \prime}$ & 0 & $\frac{2+\lambda}{2 h^{2}}$ & $\frac{-2-\lambda}{h^{2}}$ & $\frac{2+\lambda}{2 h^{2}}$ & 0 \\
\hline
\end{tabular}

Substituting (2.7) into (2.6) at $x=x_{i}$ yields equations (3.1).

$$
\begin{aligned}
& \sum_{i=-3}^{n-1} \alpha_{i}\left[E_{i}^{\prime \prime}\left(x_{i}, \lambda_{1}\right)+a_{1}\left(x_{i}\right) E_{i}^{\prime}\left(x_{i}, \lambda_{1}\right)+a_{2}\left(x_{i}\right) E_{i}\left(x_{i}, \lambda_{1}\right)\right] \\
& \quad+\sum_{i=-3}^{n-1} \beta_{i}\left[a_{3}\left(x_{i}\right) E_{i}^{\prime \prime}\left(x_{i}, \lambda_{2}\right)+a_{4}\left(x_{i}\right) E_{i}^{\prime}\left(x_{i}, \lambda_{2}\right)+a_{5}\left(x_{i}\right) E_{i}\left(x_{i}, \lambda_{2}\right)\right]=f_{1}\left(x_{i}\right), i=0,1, \ldots, n, \\
& \sum_{i=-3}^{n-1} \beta_{i}\left[E_{i}^{\prime \prime}\left(x_{i}, \lambda_{2}\right)+b_{1}\left(x_{i}\right) E_{i}^{\prime}\left(x_{i}, \lambda_{2}\right)+b_{2}\left(x_{i}\right) E_{i}\left(x_{i}, \lambda_{2}\right)\right] \\
& \quad+\sum_{i=-3}^{n-1} \alpha_{i}\left[b_{3}\left(x_{i}\right) E_{i}^{\prime \prime}\left(x_{i}, \lambda_{1}\right)+b_{4}\left(x_{i}\right) E_{i}^{\prime}\left(x_{i}, \lambda_{1}\right)+b_{5}\left(x_{i}\right) E_{i}\left(x_{i}, \lambda_{1}\right)\right]=f_{2}\left(x_{i}\right), i=0,1, \ldots, n, \\
& \sum_{i=-3}^{n-1} \alpha_{i} E_{i}\left(x, \lambda_{1}\right)=0, \quad x=0,1, \\
& \sum_{i=-3}^{n-1} \beta_{i} E_{i}\left(x, \lambda_{2}\right)=0, \quad x=0,1 .
\end{aligned}
$$

These equations produce a system of linear equations consisting of $2(n+3)$ equations with $2(n+3)$ 
unknowns, $\alpha_{-3}, \alpha_{-2}, \ldots, \alpha_{n-1}, \beta_{-3}, \beta_{-2,}, \beta_{n-1}$. This system can be written as a matrix vector

$$
\mathrm{QM}=\mathrm{R},
$$

where $M=\left[\alpha_{-3}, \alpha_{-2}, \ldots, \alpha_{n-1}, \beta_{-3}, \beta_{-2}, \ldots, \beta_{n-1}\right]^{\top}, R=\left[0, f_{1}\left(x_{0}\right), \ldots, f_{1}\left(x_{n}\right), 0,0, f_{2}\left(x_{0}\right), \ldots, f_{2}\left(x_{n}\right), 0\right]^{\top}$, and $Q$ is a $2(n+3) \times 2(n+3)$ matrix given by

$$
Q=\left(\begin{array}{ccc}
H_{1} & \mid & H_{2} \\
\cdots & \cdots & \cdots \\
H_{4} & \mid & H_{3}
\end{array}\right) .
$$

The four sub-matrices $\mathrm{H}_{1}, \mathrm{H}_{2}, \mathrm{H}_{3}$, and $\mathrm{H}_{4}$ can be written as the following.

$$
\begin{aligned}
& \mathrm{H}_{1}=\left(\begin{array}{ccccccc}
\frac{4-\lambda_{1}}{24} & \frac{8+\lambda_{1}}{12} & \frac{4-\lambda_{1}}{24} & 0 & \cdots & 0 & 0 \\
\epsilon_{1}\left(x_{0}\right) & \rho_{1}\left(x_{0}\right) & \tau_{1}\left(x_{0}\right) & 0 & \cdots & 0 & 0 \\
0 & \epsilon_{1}\left(x_{1}\right) & \rho_{1}\left(x_{1}\right) & \tau_{1}\left(x_{1}\right) & 0 & \cdots & 0 \\
\vdots & \vdots & \vdots & \vdots & \vdots & \vdots & \vdots \\
0 & \cdots & 0 & 0 & \epsilon_{1}\left(x_{\mathfrak{n}}\right) & \rho_{1}\left(x_{\mathfrak{n}}\right) & \tau_{1}\left(x_{\mathfrak{n}}\right) \\
\cdot & . & . & . & \frac{4-\lambda_{1}}{24} & \frac{8+\lambda_{1}}{12} & \frac{4-\lambda_{1}}{24}
\end{array}\right)_{(\mathrm{n}+3) \times(n+3)} \\
& \mathrm{H}_{2}=\left(\begin{array}{ccccccc}
0 & 0 & 0 & 0 & \cdots & 0 & 0 \\
\epsilon_{2}\left(x_{0}\right) & \rho_{2}\left(x_{0}\right) & \tau_{2}\left(x_{0}\right) & 0 & \cdots & 0 & 0 \\
0 & \epsilon_{2}\left(x_{1}\right) & \rho_{2}\left(x_{1}\right) & \tau_{2}\left(x_{1}\right) & 0 & \cdots & 0 \\
\vdots & \vdots & \vdots & \vdots & \vdots & \vdots & \vdots \\
0 & \cdots & 0 & 0 & \epsilon_{2}\left(x_{n}\right) & \rho_{2}\left(x_{n}\right) & \tau_{2}\left(x_{n}\right) \\
\cdot & \cdot & \cdot & \cdot & 0 & 0 & 0
\end{array}\right)_{(n+3) \times(n+3)}, \\
& \mathrm{H}_{3}=\left(\begin{array}{ccccccc}
\frac{4-\lambda_{2}}{24} & \frac{8+\lambda_{2}}{12} & \frac{4-\lambda_{2}}{24} & 0 & \cdots & 0 & 0 \\
\epsilon_{3}\left(x_{0}\right) & \rho_{3}\left(x_{0}\right) & \tau_{3}\left(x_{0}\right) & 0 & \cdots & 0 & 0 \\
0 & \epsilon_{3}\left(x_{1}\right) & \rho_{3}\left(x_{1}\right) & \tau_{3}\left(x_{1}\right) & 0 & \cdots & 0 \\
\vdots & \vdots & \vdots & \vdots & \vdots & \vdots & \vdots \\
0 & \cdots & 0 & 0 & \epsilon_{3}\left(x_{\mathfrak{n}}\right) & \rho_{3}\left(x_{\mathfrak{n}}\right) & \tau_{3}\left(x_{\mathfrak{n}}\right) \\
. & . & . & . & \frac{4-\lambda_{2}}{24} & \frac{8+\lambda_{2}}{12} & \frac{4-\lambda_{2}}{24}
\end{array}\right)_{(\mathrm{n}+3) \times(\mathrm{n}+3)} \\
& \mathrm{H}_{4}=\left(\begin{array}{ccccccc}
0 & 0 & 0 & 0 & \cdots & 0 & 0 \\
\epsilon_{4}\left(x_{0}\right) & \rho_{4}\left(x_{0}\right) & \tau_{4}\left(x_{0}\right) & 0 & \cdots & 0 & 0 \\
0 & \epsilon_{4}\left(x_{1}\right) & \rho_{4}\left(x_{1}\right) & \tau_{4}\left(x_{1}\right) & 0 & \cdots & 0 \\
\vdots & \vdots & \vdots & \vdots & \vdots & \vdots & \vdots \\
0 & \cdots & 0 & 0 & \epsilon_{4}\left(x_{n}\right) & \rho_{4}\left(x_{n}\right) & \tau_{4}\left(x_{n}\right) \\
\cdot & \cdot & \cdot & \cdot & 0 & 0 & 0
\end{array}\right)_{(n+3) \times(n+3)}
\end{aligned}
$$

The input of $H_{1}, H_{2}, H_{3}$, and $H_{4}$ are given below for $i=0,1, \ldots, n$.

$$
\begin{aligned}
& \epsilon_{1}\left(x_{i}\right)=\frac{2+\lambda_{1}}{2 h^{2}}-a_{1}\left(x_{i}\right) \frac{1}{2 h}+a_{2}\left(x_{i}\right) \frac{4-\lambda_{1}}{24}, \\
& \epsilon_{2}\left(x_{i}\right)=a_{3}\left(x_{i}\right) \frac{2+\lambda_{2}}{2 h^{2}}-a_{4}\left(x_{i}\right) \frac{1}{2 h}+a_{5}\left(x_{i}\right) \frac{4-\lambda_{2}}{24}, \\
& \epsilon_{3}\left(x_{i}\right)=\frac{2+\lambda_{2}}{2 h^{2}}-b_{1}\left(x_{i}\right) \frac{1}{2 h}+b_{2}\left(x_{i}\right) \frac{4-\lambda_{2}}{24}, \\
& \epsilon_{4}\left(x_{i}\right)=b_{3}\left(x_{i}\right) \frac{2+\lambda_{1}}{2 h^{2}}-b_{4}\left(x_{i}\right) \frac{1}{2 h}+b_{5}\left(x_{i}\right) \frac{4-\lambda_{1}}{24},
\end{aligned}
$$




$$
\begin{aligned}
& \rho_{1}\left(x_{i}\right)=\frac{-2-\lambda_{1}}{h^{2}}+a_{1}\left(x_{i}\right) \frac{0}{h}+a_{2}\left(x_{i}\right) \frac{8+\lambda_{1}}{12}, \\
& \rho_{2}\left(x_{i}\right)=a_{3}\left(x_{i}\right) \frac{-2-\lambda_{2}}{h^{2}}+a_{4}\left(x_{i}\right) \frac{0}{h}+a_{5}\left(x_{i}\right) \frac{8+\lambda_{2}}{12}, \\
& \rho_{3}\left(x_{i}\right)=\frac{-2-\lambda_{2}}{h^{2}}+b_{1}\left(x_{i}\right) \frac{0}{h}+b_{2}\left(x_{i}\right) \frac{8+\lambda_{2}}{12} \\
& \rho_{4}\left(x_{i}\right)=b_{3}\left(x_{i}\right) \frac{-2-\lambda_{1}}{h^{2}}+b_{4}\left(x_{i}\right) \frac{0}{h}+b_{5}\left(x_{i}\right) \frac{8+\lambda_{1}}{12}, \\
& \tau_{1}\left(x_{i}\right)=\frac{2+\lambda_{1}}{2 h^{2}}+a_{1}\left(x_{i}\right) \frac{1}{2 h}+a_{2}\left(x_{i}\right) \frac{4-\lambda_{1}}{24}, \\
& \tau_{2}\left(x_{i}\right)=a_{3}\left(x_{i}\right) \frac{2+\lambda_{2}}{2 h^{2}}+a_{4}\left(x_{i}\right) \frac{1}{2 h}+a_{5}\left(x_{i}\right) \frac{4-\lambda_{2}}{24}, \\
& \tau_{3}\left(x_{i}\right)=\frac{2+\lambda_{2}}{2 h^{2}}+b_{1}\left(x_{i}\right) \frac{1}{2 h}+b_{2}\left(x_{i}\right) \frac{4-\lambda_{2}}{24}, \\
& \tau_{4}\left(x_{i}\right)=b_{3}\left(x_{i}\right) \frac{2+\lambda_{1}}{2 h^{2}}+b_{4}\left(x_{i}\right) \frac{1}{2 h}+b_{5}\left(x_{i}\right) \frac{4-\lambda_{1}}{24} .
\end{aligned}
$$

To find the values of $\alpha_{i}$ 's and $\beta_{i}$ 's in terms of $\lambda_{1}$ and $\lambda_{2}$, we can use equation (3.2) by taking $M=Q^{-1} R$. Lastly, the values of $\lambda_{1}$ and $\lambda_{2}$ can be determined by optimizing the one norm as discussed in [17]. Hence, the approximate analytical solution can be generated.

\section{Truncation error}

The truncation error for the system in (2.4) has already been calculated in [17] to be of order $h^{2}$. The errors are also dependent on the values of $\lambda_{1}$ and $\lambda_{2}$.

\section{Results and discussions}

A number of examples are solved using ECBSM and the results are compared with the results of He's homotopy perturbation, Laplace homotopy analysis, variational iteration, homotopy perturbationreproducing kernel, reproducing kernel, sinc collocation, Chebyshev finite difference, spline collocation, and cubic B-spline scaling methods $[9,11,13,14,20,21,23,25,26]$. Calculations were carried out using Wolfram Mathematica 10.2 with Intel(R) Core(TM) i7 CPU 2.4GHz processor, 8.00GB RAM. The results can only be generated for $n \leqslant 10$ due to the computational limit of the computer. Numerical errors are calculated using infinite and two norms, as respectively follows:

$$
\begin{aligned}
& \mathrm{L}_{\infty}=\max _{i}\left|\mathrm{u}\left(\mathrm{x}_{\mathrm{i}}\right)-\mathrm{U}\left(\mathrm{x}_{\mathrm{i}}\right)\right| \quad \text { or } \quad \mathrm{L}_{\infty}=\max _{i}\left|v\left(\mathrm{x}_{\mathrm{i}}\right)-\mathrm{V}\left(\mathrm{x}_{\mathrm{i}}\right)\right| \text {, } \\
& \mathrm{L}_{2}=\sqrt{\sum_{i=1}^{n}\left(u\left(x_{i}\right)-\mathrm{U}\left(x_{i}\right)\right)^{2}} \quad \text { or } \quad L_{2}=\sqrt{\sum_{i=1}^{n}\left(v\left(x_{i}\right)-V\left(x_{i}\right)\right)^{2}} .
\end{aligned}
$$

Example 5.1. Consider the system of nonlinear equation [20],

$$
\left\{\begin{array}{l}
u^{\prime \prime}(x)-x v^{\prime}(x)+u(x)=f_{1}(x), \\
v^{\prime \prime}(x)+x u^{\prime}(x)+u(x) v(x)=f_{2}(x), \\
u(0)=u(1)=0, v(0)=v(1)=0,
\end{array}\right.
$$

where $0 \leqslant x \leqslant 1, f_{1}(x)=x^{3}-2 x^{2}+6 x$, and $f_{2}(x)=x^{5}-x^{4}+2 x^{3}+x^{2}-x+2$. The exact solutions are $u(x)=x^{3}-x$ and $v(x)=x^{2}-x$.

Table 2 displays the optimized values of $\lambda_{1}$ and $\lambda_{2}$ for $n=3,5,7$ and 9 together with their corresponding computational time and norms. The numerical results for Example 5.1 for $n=5$ are shown in Table 3 and compared with spline collocation method [20]. However, the results of He's and Laplace 
homotopy methods $[23,25]$ cannot be compared with the results of ECBSM because the solutions are of different class. The former produce solutions from the truncation of series whereas the latter depends on the number of domain partition. However, for $n=5$, the results of ECBSM are comparable with He's and Laplace homotopy methods. From the tables, ECBSM produces better approximation than the other methods. The plots of the numerical and exact solutions are shown in Figures 1 and 2.

Table 2: The optimized values of $\lambda_{1}$ and $\lambda_{2}$ in the last iteration, computational time and norms for $n=3,5,7$, and 9 .

\begin{tabular}{cccccc}
\hline $\mathrm{n}$ & $\lambda_{1}$ & $\lambda_{2}$ & Computational timess) & $\mathrm{L}_{\infty}$ & $\mathrm{L}_{2}$ \\
\hline 3 & 0.00000 & 0.00000 & $4.09603 \mathrm{E}-01$ & $7.21645 \mathrm{E}-12$ & $1.07855 \mathrm{E}-11$ \\
5 & $-1.30927 \mathrm{E}-13$ & $-8.81209 \mathrm{E}-14$ & $5.46864 \mathrm{E}+00$ & $2.54089 \mathrm{E}-11$ & $4.34444 \mathrm{E}-11$ \\
7 & $-8.18702 \mathrm{E}-15$ & $-5.47207 \mathrm{E}-15$ & $4.36838 \mathrm{E}+01$ & $1.68754 \mathrm{E}-10$ & $2.25381 \mathrm{E}-10$ \\
9 & $-7.00458 \mathrm{E}-14$ & $-4.67703 \mathrm{E}-14$ & $2.56199 \mathrm{E}+02$ & $3.06838 \mathrm{E}-10$ & $4.40696 \mathrm{E}-10$ \\
\hline
\end{tabular}

Table 3: Absolute errors and norms of spline collocation approach [20] and ECBSM for Example 5.1 with $n=5$ for $u(x)$ and $v(x)$.

\begin{tabular}{|c|c|c|c|c|c|c|}
\hline \multirow[t]{2}{*}{$x$} & \multicolumn{2}{|c|}{$\begin{array}{l}\text { spline collocation } \\
\text { approach }\end{array}$} & \multicolumn{2}{|c|}{$\begin{array}{c}\text { ECBSM } \\
\left(\lambda_{1}=\lambda_{2}=0\right)\end{array}$} & \multicolumn{2}{|c|}{$\begin{array}{c}\text { ECBSM } \\
\left(\lambda_{1}=-1.30927 \mathrm{E}-13, \lambda_{2}=-8.81209 \mathrm{E}-14\right)\end{array}$} \\
\hline & $u(x)$ & $v(x)$ & $u(x)$ & $v(x)$ & $u(x)$ & $v(x)$ \\
\hline 0.2 & 0.00 & $2.2 E-09$ & $4.7 E-10$ & $1.6 \mathrm{E}-10$ & $1.5 \mathrm{E}-11$ & $6.6 \mathrm{E}-12$ \\
\hline 0.4 & 0.00 & $4.3 E-09$ & $5.1 E-10$ & $2.7 E-10$ & $2.5 E-11$ & $9.7 E-12$ \\
\hline 0.6 & $3.0 E-10$ & $5.4 \mathrm{E}-09$ & $2.4 \mathrm{E}-10$ & $2.3 E-10$ & $2.4 \mathrm{E}-11$ & $1.0 \mathrm{E}-11$ \\
\hline 0.8 & $3.0 E-10$ & $3.9 E-09$ & $1.9 E-10$ & $1.4 \mathrm{E}-10$ & $6.8 E-12$ & $1.2 \mathrm{E}-11$ \\
\hline $\mathrm{L}_{\infty}$ & $3.0 \mathrm{E}-10$ & $5.4 \mathrm{E}-09$ & $5.1 E-10$ & $2.7 E-10$ & $2.5 \mathrm{E}-11$ & $1.2 \mathrm{E}-11$ \\
\hline $\mathrm{L}_{2}$ & $4.2 E-10$ & $8.2 E-09$ & $7.6 E-10$ & $4.1 E-10$ & $3.8 \mathrm{E}-11$ & $2.0 \mathrm{E}-11$ \\
\hline
\end{tabular}

Table 4: Norms of He's homotopy [25], Laplace homotopy [23], and ECBSM for Example 5.1 with $\mathrm{n}=5 \mathrm{for} u(x)$ and $v(x)$.

\begin{tabular}{ccccc}
\hline$x$ & \multicolumn{2}{c}{$u(x)$} & \multicolumn{2}{c}{$v(x)$} \\
\hline & $\mathrm{L}_{\infty}$ & $\mathrm{L}_{2}$ & $\mathrm{~L}_{\infty}$ & $\mathrm{L}_{2}$ \\
\hline ECBSM $(n=5)$ & $2.5 \mathrm{E}-11$ & $3.8 \mathrm{E}-11$ & $1.2 \mathrm{E}-11$ & $2.0 \mathrm{E}-11$ \\
He's homotopy & $7.2 \mathrm{E}-03$ & $1.2 \mathrm{E}-02$ & $9.4 \mathrm{E}-03$ & $1.4 \mathrm{E}-02$ \\
Laplace homotopy & $9.2 \mathrm{E}-06$ & $1.5 \mathrm{E}-05$ & $5.5 \mathrm{E}-05$ & $8.7 \mathrm{E}-05$ \\
\hline
\end{tabular}

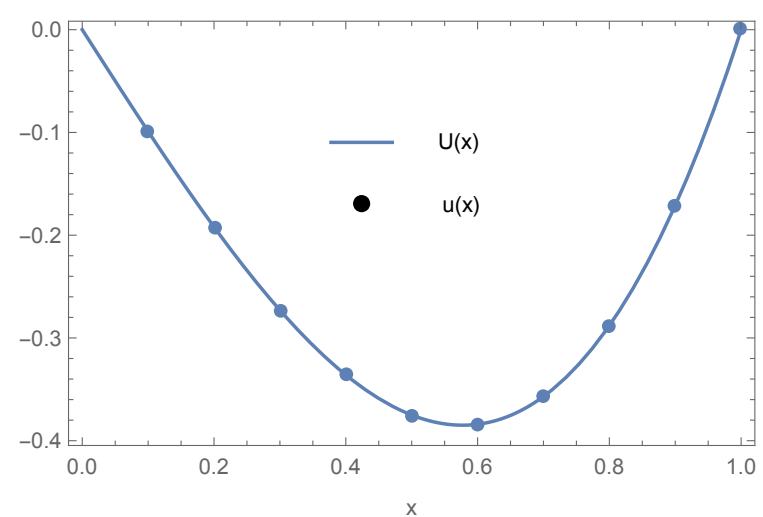

Figure 1: Numerical solution $\mathrm{U}(\mathrm{x})$ and exact solution $\mathrm{u}(\mathrm{x})$ for Example 5.1 using ECBSM with $\lambda_{1}=-1.30927 \mathrm{E}-13$, $\lambda_{2}=-8.81209 \mathrm{E}-14$, and $\mathrm{n}=5$.

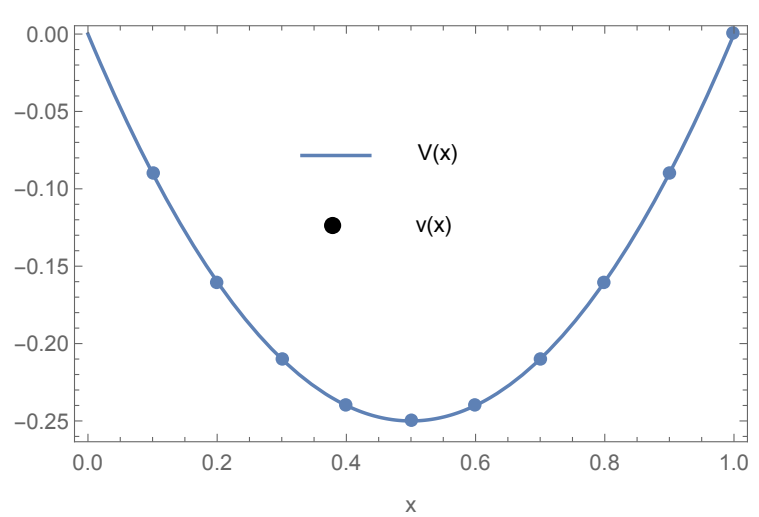

Figure 2: Numerical solution $\mathrm{V}(\mathrm{x})$ and exact solution $v(x)$ for Example 5.1 using ECBSM with $\lambda_{1}=-1.30927 \mathrm{E}-13$, $\lambda_{2}=-8.81209 \mathrm{E}-14$, and $\mathrm{n}=5$. 
Example 5.2. Consider the system of nonlinear equation includes the nonlinear $u^{\prime} v^{\prime}$-term [20].

$$
\left\{\begin{array}{l}
3 u^{\prime \prime}(x)+3 x u(x)+3 v(x)-u^{\prime}(x) v^{\prime}(x)=f_{1}(x), \\
v^{\prime \prime}(x)+x^{3} u^{\prime}(x)+x^{2} u(x)-\frac{x}{2} u^{\prime}(x) v^{\prime}(x)=f_{2}(x) \\
u(0)=0, u(1)=3, v(0)=v(1)=0
\end{array}\right.
$$

where $0 \leqslant x \leqslant 1, f_{1}(x)=8-x$, and $f_{2}(x)=x^{3}+x^{2}+7 x$. The exact solutions are $u(x)=x^{2}+2 x$, and $v(x)=x^{3}-x$.

Table 5 displays the optimized values of $\lambda_{1}$ and $\lambda_{2}$ for $n=3,5,7$ and 9 together with their corresponding computational time and norms. The numerical results for Example 5.2 for $n=5$ are shown in Table 6 and compared with spline collocation method [20]. From the tables, ECBSM produces better approximation than the other methods. The plots of the numerical and exact solutions are shown in Figures 3 and 4.

Table 5: The optimized values of $\lambda_{1}$ and $\lambda_{2}$ in the last iteration, computational time and norms for $n=3,5,7$, and 9 .

\begin{tabular}{cccccc}
\hline $\mathrm{n}$ & $\lambda_{1}$ & $\lambda_{2}$ & Computational time(s) & $\mathrm{L}_{\infty}$ & $\mathrm{L}_{2}$ \\
\hline 3 & $-3.08195 \mathrm{E}-15$ & $-3.08432 \mathrm{E}-15$ & $6.46373 \mathrm{E}-01$ & $1.28786 \mathrm{E}-11$ & $1.00254 \mathrm{E}-10$ \\
5 & $-1.48737 \mathrm{E}-15$ & $-7.01692 \mathrm{E}-15$ & $7.20707 \mathrm{E}+00$ & $7.77156 \mathrm{E}-11$ & $1.03060 \mathrm{E}-10$ \\
7 & $-4.37203 \mathrm{E}-20$ & $-4.46526 \mathrm{E}-20$ & $5.08717 \mathrm{E}+01$ & $8.12683 \mathrm{E}-11$ & $9.20049 \mathrm{E}-10$ \\
9 & $-9.85542 \mathrm{E}-13$ & $-1.07684 \mathrm{E}-12$ & $2.83140 \mathrm{E}+02$ & $2.76223 \mathrm{E}-10$ & $5.66209 \mathrm{E}-09$ \\
\hline
\end{tabular}

Table 6: Absolute errors and norms of spline collocation approach [20] and ECBSM for Example 5.2 with $n=5$ for $u(x)$ and $v(\mathrm{x})$.

\begin{tabular}{|c|c|c|c|c|c|c|}
\hline \multirow[t]{2}{*}{$x$} & \multicolumn{2}{|c|}{$\begin{array}{l}\text { spline collocation } \\
\text { approach }\end{array}$} & \multicolumn{2}{|c|}{$\begin{array}{c}\text { ECBSM } \\
\left(\lambda_{1}=\lambda_{2}=0\right)\end{array}$} & \multicolumn{2}{|c|}{$\begin{array}{c}\text { ECBSM } \\
\left(\lambda_{1}=-1.48737 \mathrm{E}-15, \lambda_{2}=-2.24845 \mathrm{E}-15\right)\end{array}$} \\
\hline & $u(x)$ & $v(x)$ & $u(x)$ & $v(x)$ & $u(x)$ & $v(x)$ \\
\hline 0.2 & $3.8 \mathrm{E}-08$ & $2.3 \mathrm{E}-08$ & $1.5 \mathrm{E}-09$ & $1.1 \mathrm{E}-10$ & $1.7 \mathrm{E}-11$ & $6.7 \mathrm{E}-12$ \\
\hline 0.4 & $5.3 \mathrm{E}-08$ & $4.3 \mathrm{E}-08$ & $3.4 \mathrm{E}-09$ & $3.9 \mathrm{E}-10$ & $2.2 \mathrm{E}-12$ & $3.9 E-12$ \\
\hline 0.6 & $6.3 \mathrm{E}-08$ & $6.2 \mathrm{E}-08$ & $2.2 \mathrm{E}-09$ & $2.2 \mathrm{E}-10$ & $4.4 \mathrm{E}-11$ & $7.8 \mathrm{E}-12$ \\
\hline 0.8 & $6.8 \mathrm{E}-08$ & $7.6 \mathrm{E}-08$ & $3.4 \mathrm{E}-09$ & $3.8 \mathrm{E}-10$ & $4.9 \mathrm{E}-11$ & $7.8 \mathrm{E}-11$ \\
\hline $\mathrm{L}_{\infty}$ & $6.8 \mathrm{E}-08$ & $7.6 \mathrm{E}-08$ & $3.4 \mathrm{E}-09$ & $4.9 \mathrm{E}-10$ & $4.9 \mathrm{E}-11$ & $7.8 \mathrm{E}-11$ \\
\hline $\mathrm{L}_{2}$ & $1.1 \mathrm{E}-07$ & $1.1 \mathrm{E}-07$ & $5.5 \mathrm{E}-09$ & $7.1 \mathrm{E}-10$ & $6.8 \mathrm{E}-11$ & $7.8 \mathrm{E}-11$ \\
\hline
\end{tabular}

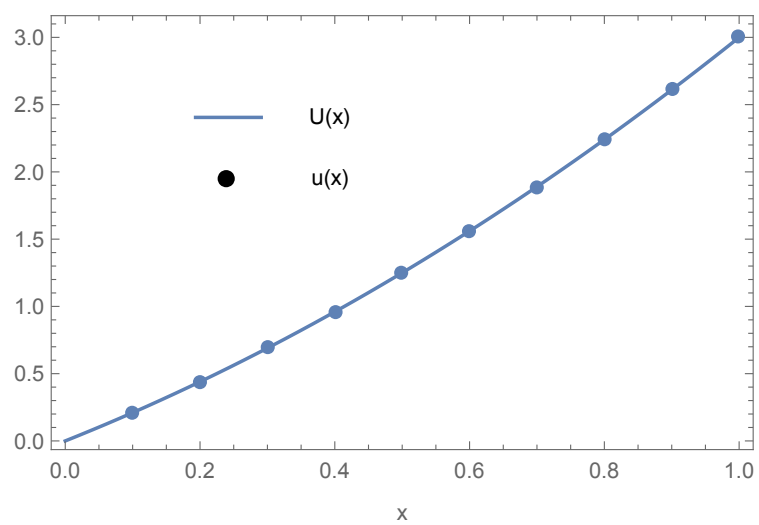

Figure 3: Numerical solution $\mathrm{U}(\mathrm{x})$ and exact solution $\mathrm{u}(\mathrm{x})$ for Example 5.2 using ECBSM with $\lambda_{1}=-1.48737 \mathrm{E}-15$, $\lambda_{2}=-2.24845 \mathrm{E}-15$, and $\mathrm{n}=5$.

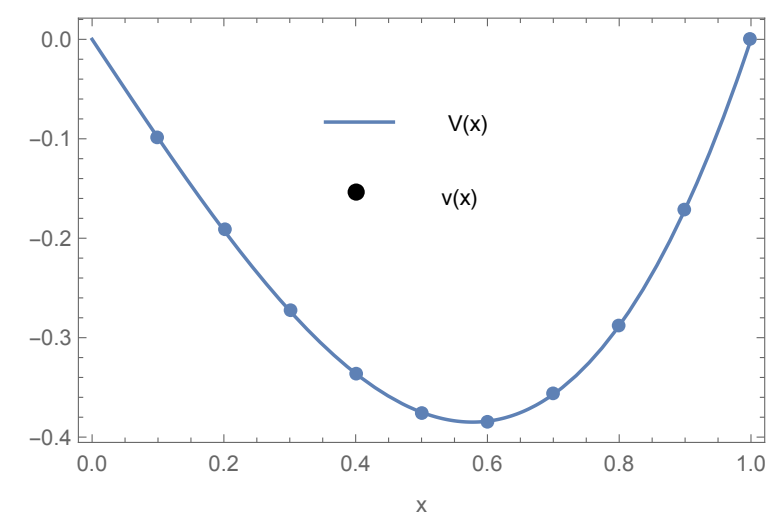

Figure 4: Numerical solution $\mathrm{V}(\mathrm{x})$ and exact solution $v(\mathrm{x})$ for Example 5.2 using ECBSM with $\lambda_{1}=-1.48737 \mathrm{E}-15$, $\lambda_{2}=-2.24845 \mathrm{E}-15$, and $\mathrm{n}=5$. 
Example 5.3. Consider a system of nonlinear equation [14],

$$
\left\{\begin{array}{l}
u^{\prime \prime}(x)+x u^{\prime}(x)+\cos (\pi x) v^{\prime}(x)=f_{1}(x) \\
v^{\prime \prime}(x)+x u^{\prime}(x)+x u^{2}(x)=f_{2}(x) \\
u(0)=u(1)=0, v(0)=v(1)=0
\end{array}\right.
$$

where $0 \leqslant x \leqslant 1, f_{1}(x)=\sin (x)+\left(x^{2}-x+2\right) \cos (x)+(1-2 x) \cos (\pi x)$, and $f_{2}(x)=-2+x \sin (x)+x(x-$ $1)^{2} \sin ^{2}(x)+\left(x^{2}-x\right) \cos (x)$. The exact solutions are $u(x)=(x-1) \sin (x)$, and $v(x)=x-x^{2}$.

Table 7 displays the optimized values of $\lambda_{1}$ and $\lambda_{2}$ for $n=3,5,7$ and 10 together with their corresponding computational time and norms. The numerical results for Example 5.3 for $n=10$ are shown in Tables 8 and 9 and compared with VIM and homotopy perturbation-reproducing kernel methods [14, 21]. From the tables, ECBSM produces better approximation than the other methods. The plots of the numerical and exact solutions are shown in Figures 5 and 6.

Table 7: The optimized values of $\lambda_{1}$ and $\lambda_{2}$ in the last iteration, computational time and norms for $n=3,5,7$, and 10 .

\begin{tabular}{cccccc}
\hline $\mathrm{n}$ & $\lambda_{1}$ & $\lambda_{2}$ & Computational time $(\mathrm{s})$ & $\mathrm{L}_{\infty}$ & $\mathrm{L}_{2}$ \\
\hline 3 & $-3.57648 \mathrm{E}-02$ & $3.07651 \mathrm{E}-05$ & $4.26617 \mathrm{E}-01$ & $3.04790 \mathrm{E}-05$ & $3.79185 \mathrm{E}-05$ \\
5 & $-1.27073 \mathrm{E}-02$ & $2.98753 \mathrm{E}-06$ & $2.61117 \mathrm{E}+00$ & $3.42265 \mathrm{E}-06$ & $4.37469 \mathrm{E}-06$ \\
7 & $-6.48231 \mathrm{E}-03$ & $6.63778 \mathrm{E}-07$ & $1.76063 \mathrm{E}+01$ & $2.37250 \mathrm{E}-06$ & $3.15425 \mathrm{E}-06$ \\
10 & $-3.16155 \mathrm{E}-03$ & $1.67076 \mathrm{E}-07$ & $2.75975 \mathrm{E}+02$ & $1.23232 \mathrm{E}-06$ & $1.67593 \mathrm{E}-06$ \\
\hline
\end{tabular}

Table 8: Absolute errors and norms of ECBSM for Example 5.4 with $n=10$ for $v(x)$.

\begin{tabular}{ccccc}
\hline & VIM & HP-RKM & $\begin{array}{c}\text { ECBSM } \\
\left(\lambda_{1}=\lambda_{2}=0\right)\end{array}$ & $\left(\lambda_{1}=-3.16155 \mathrm{E}-03, \lambda_{2}=1.67076 \mathrm{E}-07\right)$ \\
\hline 0.1 & $3.0 \mathrm{E}-04$ & $4.4 \mathrm{E}-05$ & $1.4 \mathrm{E}-04$ & $1.2 \mathrm{E}-06$ \\
0.3 & $7.8 \mathrm{E}-03$ & $1.0 \mathrm{E}-04$ & $3.2 \mathrm{E}-04$ & $1.0 \mathrm{E}-06$ \\
0.5 & $2.7 \mathrm{E}-02$ & $1.2 \mathrm{E}-04$ & $3.6 \mathrm{E}-04$ & $1.6 \mathrm{E}-07$ \\
0.7 & $4.6 \mathrm{E}-02$ & $9.6 \mathrm{E}-05$ & $2.7 \mathrm{E}-04$ & $1.9 \mathrm{E}-07$ \\
0.9 & $3.1 \mathrm{E}-02$ & $3.8 \mathrm{E}-05$ & $1.0 \mathrm{E}-04$ & $4.5 \mathrm{E}-07$ \\
\hline $\mathrm{L}_{\infty}$ & $4.6 \mathrm{E}-02$ & $1.2 \mathrm{E}-04$ & $3.6 \mathrm{E}-04$ & $1.2 \mathrm{E}-06$ \\
$\mathrm{~L}_{2}$ & $6.2 \mathrm{E}-02$ & $1.9 \mathrm{E}-04$ & $5.8 \mathrm{E}-04$ & $1.6 \mathrm{E}-06$ \\
\hline
\end{tabular}

Table 9: Absolute errors and norms of ECBSM for Example 5.3 with $n=10$ for $v(x)$.

\begin{tabular}{ccc}
\hline & $\begin{array}{c}\mathrm{ECBSM} \\
\left(\lambda_{1}=\lambda_{2}=0\right)\end{array}$ & $\left(\lambda_{1}=-3.16155 \mathrm{E}-03, \lambda_{2}=1.67076 \mathrm{E}-07\right)$ \\
\hline 0.1 & $3.2 \mathrm{E}-06$ & $2.7 \mathrm{E}-08$ \\
0.3 & $1.3 \mathrm{E}-05$ & $7.7 \mathrm{E}-08$ \\
0.5 & $2.7 \mathrm{E}-05$ & $6.6 \mathrm{E}-08$ \\
0.7 & $3.4 \mathrm{E}-05$ & $1.6 \mathrm{E}-08$ \\
0.9 & $2.0 \mathrm{E}-05$ & $1.0 \mathrm{E}-08$ \\
\hline $\mathrm{L}_{\infty}$ & $3.4 \mathrm{E}-05$ & $7.7 \mathrm{E}-08$ \\
$\mathrm{~L}_{2}$ & $5.0 \mathrm{E}-05$ & $1.1 \mathrm{E}-07$ \\
\hline
\end{tabular}




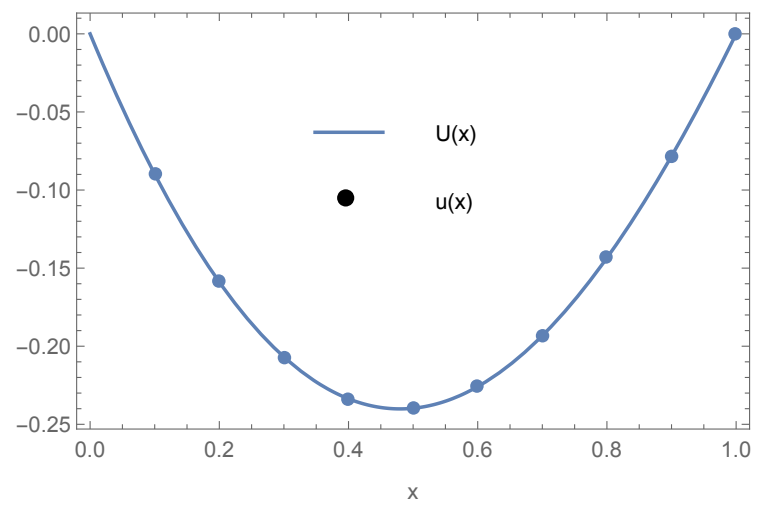

Figure 5: Numerical solution $\mathrm{U}(x)$ and exact solution $u(x)$ for Example 5.3 using ECBSM with $\lambda_{1}=-3.16155 \mathrm{E}-03$, $\lambda_{2}=1.67076 \mathrm{E}-07$, and $n=10$.

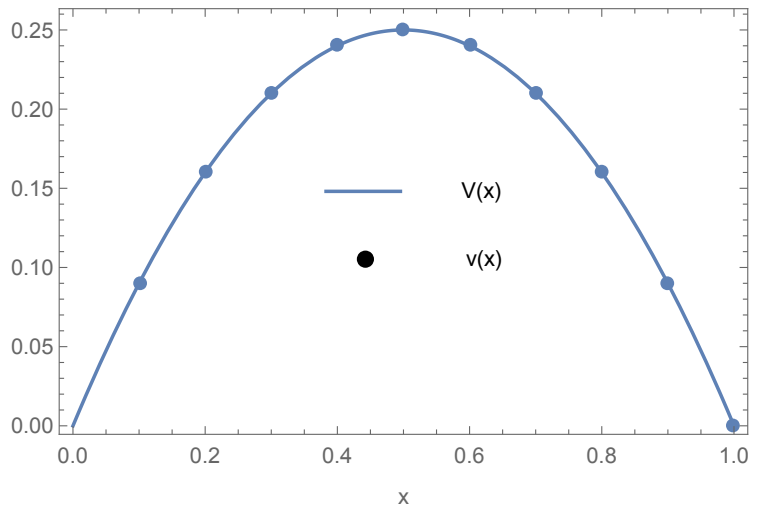

Figure 6: Numerical solution $\mathrm{V}(\mathrm{x})$ and exact solution $v(\mathrm{x})$ for Example 5.3 using ECBSM with $\lambda_{1}=-3.16155 \mathrm{E}-03$, $\lambda_{2}=1.67076 \mathrm{E}-07$, and $\mathrm{n}=10$.

Example 5.4. Consider a system of nonlinear equation [9],

$$
\left\{\begin{array}{l}
u^{\prime \prime}(x)+x u(x)+2 x v(x)+x u^{2}(x)=f_{1}(x), \\
v^{\prime \prime}(x)+v(x)+x^{2} u(x)+\sin (x) v^{2}(x)=f_{2}(x), \\
u(0)=u(1)=0, v(0)=v(1)=0,
\end{array}\right.
$$

where $0 \leqslant x \leqslant 1, f_{1}(x)=2 x \sin (\pi x)+x^{5}-2 x^{4}+x^{2}-2$, and $f_{2}(x)=x^{3}(1-x)+\left(1-\pi^{2}\right) \sin (\pi x)+$ $\sin (x) \sin ^{2}(\pi x)$. The exact solutions are $u(x)=x-x^{2}$, and $v(x)=\sin (\pi x)$.

Table 10 displays the optimized values of $\lambda_{1}$ and $\lambda_{2}$ for $n=3,5,7$ and 10 together with their corresponding computational time and norms. The numerical results for Example 5.4 for $n=10$ are shown in Tables 11 and 12 and compared with homotopy perturbation-reproducing kernel, reproducing kernel, sinc collocation, Chebyshev finite difference, and cubic B-spline scaling methods [9, 11, 13, 14, 26]. From the tables, ECBSM produces better approximation than the other methods. The plots of the numerical and exact solutions are shown in Figures 7 and 8.

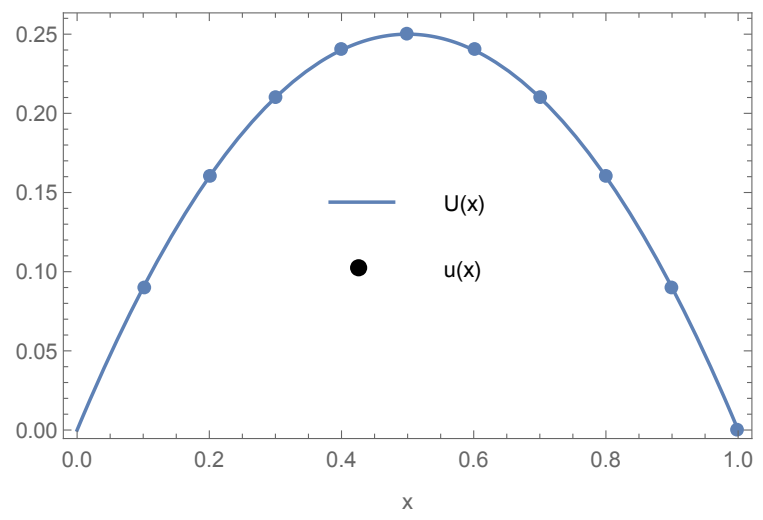

Figure 7: Numerical solution $\mathrm{U}(\mathrm{x})$ and exact solution $\mathrm{u}(\mathrm{x})$ for Example 5.4 using ECBSM with $\lambda_{1}=1.67646 \mathrm{E}-08, \lambda_{2}=$ $-1.64832 \mathrm{E}-02$, and $\mathrm{n}=10$.

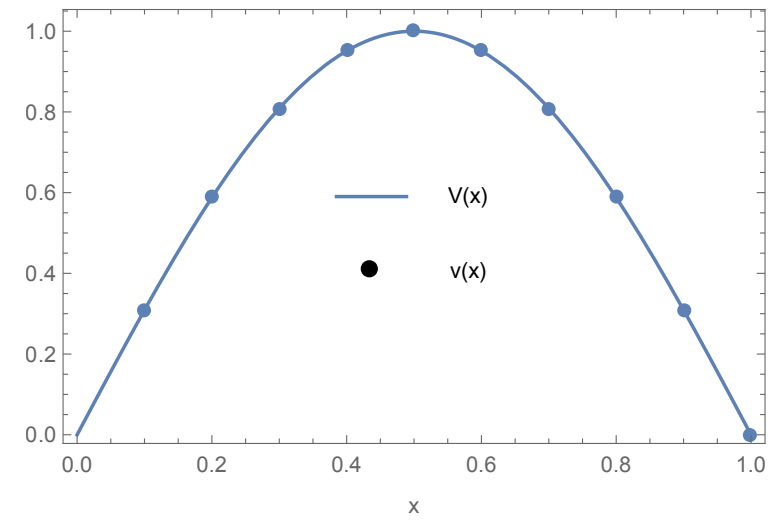

Figure 8: Numerical solution $\mathrm{V}(\mathrm{x})$ and exact solution $v(x)$ for Example 5.4 using ECBSM with $\lambda_{1}=1.67646 \mathrm{E}-08, \lambda_{2}=$ $-1.64832 \mathrm{E}-02$, and $\mathrm{n}=10$. 
Table 10: The optimized values of $\lambda_{1}$ and $\lambda_{2}$ in the last iteration, computational time and norms for $n=3,5,7$, and 10 .

\begin{tabular}{cccccc}
\hline $\mathrm{n}$ & $\lambda_{1}$ & $\lambda_{2}$ & Computational time $(\mathrm{s})$ & $\mathrm{L}_{\infty}$ & $\mathrm{L}_{2}$ \\
\hline 3 & $1.32459 \mathrm{E}-06$ & $-1.87092 \mathrm{E}-01$ & $2.37289 \mathrm{E}-01$ & $1.34147 \mathrm{E}-03$ & $2.31416 \mathrm{E}-03$ \\
5 & $3.11779 \mathrm{E}-07$ & $-6.63416 \mathrm{E}-02$ & $5.34669 \mathrm{E}+00$ & $1.93619 \mathrm{E}-04$ & $3.22282 \mathrm{E}-04$ \\
7 & $5.19719 \mathrm{E}-08$ & $-3.37116 \mathrm{E}-02$ & $2.96829 \mathrm{E}+01$ & $5.06215 \mathrm{E}-05$ & $8.21971 \mathrm{E}-05$ \\
10 & $1.67646 \mathrm{E}-08$ & $-1.64832 \mathrm{E}-02$ & $4.01543 \mathrm{E}+02$ & $1.21298 \mathrm{E}-05$ & $2.21764 \mathrm{E}-05$ \\
\hline
\end{tabular}

Table 11: Absolute errors and norms of RKM [13], sinc collocation [11], HP-RKM [14], ChFD [26], cubic B-spline scaling [9], and ECBSM for Example 5.4 with $\lambda_{1}=1.67646 \mathrm{E}-08, \lambda_{2}=-1.64832 \mathrm{E}-02$, and $n=10$ for $u(x)$.

\begin{tabular}{cccccccc}
\hline$x$ & RKM & $\begin{array}{c}\text { sinc } \\
\text { collocation }\end{array}$ & HP-RKM & ChFD & $\begin{array}{c}\text { cubic } \\
\text { B-spline } \\
\text { scaling }\end{array}$ & $\begin{array}{c}\text { ECBSM } \\
\lambda_{1}=0, \\
\lambda_{2}=0,\end{array}$ & ECBSM \\
\hline 0.08 & $5.0 \mathrm{E}-04$ & $1.4 \mathrm{E}-04$ & $7.7 \mathrm{E}-05$ & $2.8 \mathrm{E}-05$ & $2.1 \mathrm{E}-04$ & $2.2 \mathrm{E}-04$ & $2.8 \mathrm{E}-07$ \\
0.24 & $1.4 \mathrm{E}-03$ & $4.4 \mathrm{E}-05$ & $2.2 \mathrm{E}-04$ & $7.1 \mathrm{E}-05$ & $1.6 \mathrm{E}-04$ & $6.5 \mathrm{E}-04$ & $8.1 \mathrm{E}-07$ \\
0.40 & $2.1 \mathrm{E}-03$ & $6.7 \mathrm{E}-05$ & $3.3 \mathrm{E}-04$ & $1.1 \mathrm{E}-04$ & $8.5 \mathrm{E}-05$ & $9.8 \mathrm{E}-04$ & $1.2 \mathrm{E}-06$ \\
0.56 & $2.2 \mathrm{E}-03$ & $9.3 \mathrm{E}-05$ & $3.7 \mathrm{E}-04$ & $1.4 \mathrm{E}-04$ & $1.3 \mathrm{E}-04$ & $1.1 \mathrm{E}-03$ & $1.4 \mathrm{E}-06$ \\
0.72 & $1.8 \mathrm{E}-03$ & $4.9 \mathrm{E}-05$ & $3.1 \mathrm{E}-04$ & $1.3 \mathrm{E}-04$ & $8.8 \mathrm{E}-05$ & $9.4 \mathrm{E}-04$ & $1.2 \mathrm{E}-06$ \\
0.88 & $9.0 \mathrm{E}-04$ & $8.6 \mathrm{E}-05$ & $1.5 \mathrm{E}-04$ & $7.1 \mathrm{E}-05$ & $2.3 \mathrm{E}-04$ & $4.7 \mathrm{E}-04$ & $5.9 \mathrm{E}-07$ \\
0.96 & $3.0 \mathrm{E}-04$ & $7.1 \mathrm{E}-05$ & $5.4 \mathrm{E}-05$ & $2.4 \mathrm{E}-05$ & $1.3 \mathrm{E}-04$ & $1.6 \mathrm{E}-04$ & $2.0 \mathrm{E}-07$ \\
\hline $\mathrm{L}_{\infty}$ & $2.2 \mathrm{E}-03$ & $1.4 \mathrm{E}-04$ & $3.7 \mathrm{E}-04$ & $1.4 \mathrm{E}-04$ & $2.3 \mathrm{E}-04$ & $1.1 \mathrm{E}-03$ & $1.4 \mathrm{E}-06$ \\
$\mathrm{~L}_{2}$ & $3.9 \mathrm{E}-03$ & $2.2 \mathrm{E}-04$ & $6.5 \mathrm{E}-04$ & $2.5 \mathrm{E}-04$ & $4.1 \mathrm{E}-04$ & $1.9 \mathrm{E}-03$ & $2.4 \mathrm{E}-06$ \\
\hline
\end{tabular}

Table 12: Absolute errors and norms of RKM [13], sinc collocation [11], HP-RKM [14], ChFD [26], cubic B-spline scaling [9], and ECBSM for Example 5.4 with $\lambda_{1}=1.67646 \mathrm{E}-08, \lambda_{2}=-1.64832 \mathrm{E}-02$, and $n=10$ for $v(\mathrm{x})$.

\begin{tabular}{cccccccc}
\hline$x$ & RKM & $\begin{array}{c}\text { sinc } \\
\text { collocation }\end{array}$ & HP-RKM & ChFD & $\begin{array}{c}\text { cubic } \\
\text { B-spline } \\
\text { scaling }\end{array}$ & $\begin{array}{c}\text { ECBSM } \\
\lambda_{1}=0, \\
\lambda_{2}=0,\end{array}$ & ECBSM \\
\hline 0.08 & $2.0 \mathrm{E}-03$ & $2.4 \mathrm{E}-04$ & $7.1 \mathrm{E}-04$ & $4.0 \mathrm{E}-03$ & $8.1 \mathrm{E}-04$ & $2.5 \mathrm{E}-03$ & $3.6 \mathrm{E}-06$ \\
0.24 & $5.6 \mathrm{E}-03$ & $2.3 \mathrm{E}-03$ & $1.9 \mathrm{E}-03$ & $1.0 \mathrm{E}-03$ & $8.3 \mathrm{E}-04$ & $6.8 \mathrm{E}-03$ & $8.1 \mathrm{E}-06$ \\
0.40 & $7.9 \mathrm{E}-03$ & $8.9 \mathrm{E}-04$ & $2.7 \mathrm{E}-03$ & $2.2 \mathrm{E}-04$ & $7.0 \mathrm{E}-04$ & $9.5 \mathrm{E}-03$ & $1.2 \mathrm{E}-05$ \\
0.56 & $8.2 \mathrm{E}-03$ & $1.4 \mathrm{E}-03$ & $2.8 \mathrm{E}-03$ & $1.5 \mathrm{E}-03$ & $3.5 \mathrm{E}-04$ & $9.9 \mathrm{E}-03$ & $1.2 \mathrm{E}-05$ \\
0.72 & $6.5 \mathrm{E}-03$ & $3.1 \mathrm{E}-03$ & $2.2 \mathrm{E}-03$ & $1.2 \mathrm{E}-03$ & $1.7 \mathrm{E}-04$ & $7.8 \mathrm{E}-03$ & $1.0 \mathrm{E}-05$ \\
0.88 & $3.1 \mathrm{E}-03$ & $1.6 \mathrm{E}-03$ & $1.7 \mathrm{E}-03$ & $5.1 \mathrm{E}-04$ & $7.4 \mathrm{E}-04$ & $3.7 \mathrm{E}-03$ & $4.0 \mathrm{E}-06$ \\
0.96 & $1.0 \mathrm{E}-03$ & $9.8 \mathrm{E}-04$ & $3.6 \mathrm{E}-04$ & $3.7 \mathrm{E}-04$ & $4.6 \mathrm{E}-04$ & $1.3 \mathrm{E}-03$ & $1.2 \mathrm{E}-06$ \\
\hline $\mathrm{L}_{\infty}$ & $8.2 \mathrm{E}-03$ & $3.1 \mathrm{E}-03$ & $2.8 \mathrm{E}-03$ & $4.0 \mathrm{E}-03$ & $8.3 \mathrm{E}-04$ & $9.9 \mathrm{E}-03$ & $1.2 \mathrm{E}-05$ \\
$\mathrm{~L}_{2}$ & $1.5 \mathrm{E}-02$ & $4.6 \mathrm{E}-03$ & $5.2 \mathrm{E}-03$ & $4.6 \mathrm{E}-03$ & $1.7 \mathrm{E}-03$ & $1.8 \mathrm{E}-02$ & $2.2 \mathrm{E}-05$ \\
\hline
\end{tabular}

\section{Acknowledgment}

We appreciate the reviewers and editors for their careful reading, valuable comments, timely review and reply.

\section{References}

[1] N. N. Abd Hamid, A. A. Majid, A. I. M. Ismail, Extended cubic B-spline method for linear two-point boundary value problems, Sains Malaysiana, 40 (2011), 1285-1290. 1, 2, 3

[2] O. Abu Arqub, Z. Abo-Hammour, Numerical solution of systems of second-order boundary value problems using continuous genetic algorithm, Inform. Sci., 279 (2014), 396-415. 1

[3] N. R. Anakira, A. K. Alomari, A. F. Jameel, I. Hashim, Multistage optimal homotopy asymptotic method for solving initial-value problems, J. Nonlinear Sci. Appl., 9 (2016), 1826-1843. 1 
[4] N. R. Anakira, A. Jameel, A. K. Alomari, A. Saaban, M. Almahameed, I. Hashim, Approximate solutions of multipantograph type delay differential equations using multistage optimal homotopy asymptotic method, J. Math. Fundam. Sci., 50 (2018), 221-232. 1

[5] A. S. Bataineh, M. S. M. Noorani, I. Hashim, Modified homotopy analysis method for solving systems of second-order BVPs, Commun. Nonlinear Sci. Numer. Simul., 14 (2009), 430-442. 1

[6] N. Caglar, H. Caglar, B-spline method for solving linear system of second-order boundary value problems, Comput. Math. Appl., 57 (2009), 757-762. 1

[7] S. Chen, J. Hu, L. Chen, C. Wang, Existence results for n-point boundary value problem of second order ordinary differential equations, J. Comput. Appl. Math., 180 (2005), 425-432. 1

[8] X. Cheng, C. Zhong, Existence of positive solutions for a second-order ordinary differential system, J. Math. Anal. Appl., 312 (2005), 14-23. 1

[9] M. Dehghan, M. Lakestani, Numerical solution of nonlinear system of second-order boundary value problems using cubic B-spline scaling functions, Int. J. Comput. Math., 85 (2008), 1455-1461. 1, 5, 5.4, 5, 11, 12

[10] M. Dehghan, A. Nikpour, Numerical solution of the system of second-order boundary value problems using the local radial basis functions based differential quadrature collocation method, Appl. Math. Model., 37 (2013), 8578-8599. 1

[11] M. Dehghan, A. Saadatmandi, The numerical solution of a nonlinear system of second-order boundary value problems using the sinc-collocation method, Math. Comput. Modelling, 46 (2007), 1434-1441. 1, 5, 5, 11, 12

[12] M. El-Gamel, Sinc-collocation method for solving linear and nonlinear system of second-order boundary value problems, Appl. Math., 3 (2012), 1627-1633. 1

[13] F. Geng, M. Cui, Solving a nonlinear system of second order boundary value problems, J. Math. Anal. Appl., 327 (2007), 1167-1181. 1, 5, 5, 11, 12

[14] F. Geng, M. Cui, Homotopy perturbation-reproducing kernel method for nonlinear systems of second order boundary value problems, J. Comput. Appl. Math., 235 (2011), 2405-2411. 1, 5, 5.3, 5, 5, 11, 12

[15] J. Goh, A. A. Majid, A. I. M. Ismail, Extended cubic uniform B-spline for a class of singular boundary value problems, ScienceAsia, 37 (2011), 79-82. 1, 2

[16] R. Hatamleh, Multidimensional triangular models for the system of linear operator with given properties of commutators, Ph. D. Dissertation, Kharkove, (1995). 1

[17] A. S. Heilat, N. N. A. Hamid, A. I. M. Ismail, Extended cubic B-spline method for solving a linear system of second-order boundary value problems, SpringerPlus, 5 (2016), 18 pages. 1, 3, 4

[18] A. S. Heilat, A. I. M. Ismail, Hybrid cubic b-spline method for solving Non-linear two-point boundary value problems, Int. J. Pure Appl. Math., 110 (2016), 369-381. 1

[19] P. Kang, Z. L. Wei, Three positive solutions of singular nonlocal boundary value problems for systems of nonlinear secondorder ordinary differential equations, Nonlinear Anal., 70 (2009), 444-451. 1

[20] S. A. Khuri, A. Sayfy, Spline collocation approach for the numerical solution of a generalized system of second-order boundary-value problems, Appl. Math. Sci. (Ruse), 3 (2009), 2227-2239. 1, 5, 5.1, 5, 3, 5.2, 5, 6

[21] J. F. Lu, Variational iteration method for solving a nonlinear system of second-order boundary value problems, Comput. Math. Appl., 54 (2007), 1133-1138. 1, 5, 5

[22] A. K. Nasab, Z. P. Atabakan, A. I. Ismail, An Accurate Numerical Algorithm for Solving Singular and Nonsingular System of Initial Value Problems on Large Interval, Mediterr. J. Math., 13 (2016), 5033-5051. 1

[23] O. M. Ogunlaran, A. T. Ademola, On the Laplace homotopy analysis method for a nonlinear system of second-order boundary value problems, Gen., 26 (2015), 11-22. 1, 5, 5, 4

[24] A. S. V. Ravi Kanth, Cubic spline polynomial for non-linear singular two-point boundary value problems, Appl. Math. Comput., 189 (2007), 2017-2022. 2

[25] A. Saadatmandi, M. Dehghan, A. Eftekhari, Application of He's homotopy perturbation method for non-linear system of second-order boundary value problems, Nonlinear Anal. Real World Appl., 10 (2009), 1912-1922. 1, 5, 5, 4

[26] A. Saadatmandi, J. A. Farsangi, Chebyshev finite difference method for a nonlinear system of second-order boundary value problems, Appl. Math. Comput., 192 (2007), 586-591. 1, 5, 5, 11, 12

[27] H. X. L. Shengjun, An Extension of the Cubic Uniform B-Spline Curve [J], J. Computer Aided Design Comput. Graphics, 5 (2003), 15 pages. 1

[28] H. B. Thompson, C. Tisdell, Boundary value problems for systems of difference equations associated with systems of second-order ordinary differential equations, Appl. Math. Lett., 15 (2002), 761-766. 1

[29] G. Xu, G.-Z. Wang, Extended cubic uniform B-spline and $\alpha$-B-spline, Acta Automat. Sinica, 34 (2008), 980-984. 1 